\title{
EXTENSION OF RANDOM CONTRACTIONS
}

\author{
J. MYJAK AND W. ZYGADLEWICZ
}

(Communicated by R. Daniel Mauldin)

\begin{abstract}
A BStRACT. Let $\Omega$ be a measurable space. Let $X$ and $Y$ be separable Hilbert spaces and let $D$ be a subset of $X$. Then every random contraction $f: \Omega \times$ $D \rightarrow Y$ can be extended to a random contraction defined on all $\Omega \times X$. This statement remains true if $\Omega$ is a complete measurable space, $X$ and $Y$ are separable metric spaces and the pair $(X, Y)$ has the Kirszbraun intersection property.
\end{abstract}

1. Introduction and notions. Let $\left\{X, d_{1}\right\}$ and $\left\{Y, d_{2}\right\}$ be metric spaces and let $f: D \rightarrow Y$ be a contraction from a nonempty subset $D$ of $X$ into $Y$, that is,

$$
d_{2}\left(f\left(x_{1}\right), f\left(x_{2}\right)\right) \leq d_{1}\left(x_{1}, x_{2}\right) \quad\left(x_{1}, x_{2} \in X\right) .
$$

The extension problem for contractions asks under what conditions can one always guarantee the existence of a contraction $\tilde{f}: X \rightarrow Y$ such that $\left.\tilde{f}\right|_{D}=f$. This problem has been studied by several authors; for a survey of basic results, see [3 and 8]. In particular, F. Valentine [9] proved that extension is always possible for a given pair $(X, Y)$ if and only if the following property holds:

KIRSZBRAUN INTERSECTION PROPERTY. For all choices $x_{i} \in X, y_{i} \in Y$ and $r_{i}>0, i \in J$ ( $J$ an arbitrary index set) such that the intersection of the balls $S\left(x_{j}, r_{j}\right)$ in $X$ is nonempty and $d_{2}\left(y_{i}, y_{j}\right) \leq d_{1}\left(x_{i}, x_{j}\right), i, j \in J$, it follows that the intersection of the balls $S\left(y_{i}, r_{i}\right)$ in $Y$ is also nonempty.

Here $S(x, r)$ denotes the closed ball about $x$ of radius $r$. Note that the Kirszbraun property (property $(\mathrm{K})$ ) is satisfied if $X$ and $Y$ are Hilbert spaces (see [3, 9]). In this paper we are concerned with the extension problem for random contractions.

Let $\Omega$ be a measurable space with $\sigma$-algebra $\mathscr{A}$ and let $D$ be a nonempty subset of $X$. A map $f: \Omega \times D \rightarrow Y$ is called a random contraction if, for every $x \in D, f(\cdot, x)$ is measurable and, for every $\omega \in \Omega, f(\omega, \cdot)$ is a contraction. Our major results state conditions under which there exists a random contraction $\tilde{f}: \Omega \times X \rightarrow Y$ such that $\left.\tilde{f}\right|_{\Omega \times D}=f$.

Ideas from the theory of measurable multifunctions play a dominant role in the proofs. Let $2^{X}$ denote the family of all nonempty subsets of $X$. A map $\Gamma: \Omega \rightarrow 2^{X}$ is said to be measurable (resp. weakly measurable) if for every closed (resp. open) subset $A$ of $X$, the set $\{\omega \in \Omega: \Gamma(\omega) \cap A \neq \varnothing\}$ belongs to $\mathscr{A}$. Note that if $X$ is a metric space, measurability implies weak measurability. If, in addition, $X$ is $\sigma$ compact, then measurability is equivalent to weak measurability. By a measurable

Received by the editors April 13, 1987.

1980 Mathematics Subject Classification (1985 Revision). Primary 54C20; Secondary 47H09.

Key words and phrases. Random contraction, random isometry, extension, multifunction, measurable selection, separable metric space, Hilbert space. 
selection of $\Gamma$ we mean a measurable function $\gamma: \Omega \rightarrow X$ such that $\gamma(\omega) \in \Gamma(\omega)$ for every $\omega \in \Omega$.

\section{Results.}

LEMMA 1. Let $\Omega$ be a measurable space and let $X$ be a separable Hilbert space. Let $f_{i}: \Omega \rightarrow X$ and $\rho_{i}: \Omega \rightarrow[0,+\infty), i=1,2, \ldots$, be measurable functions such that for every $\omega \in \Omega$,

$$
\Sigma(\omega)=\bigcap_{i \geq 1} S\left(f_{i}(\omega) \rho_{i}(\omega)\right) \neq \varnothing .
$$

Then the multifunction $\Gamma: \Omega \rightarrow 2^{X}$ given by (1) is weakly measurable.

Proof. By [1, Theorem III.4.1] the multifunctions $S\left(f_{i}(\cdot), \rho_{i}(\cdot)\right), i \in \mathbf{N}$, are weakly measurable. Clearly they are also weakly measurable and, in addition, compact valued, with respect to the weak topology of $X$. Thus, by virtue of $[5$, Theorem 4.1] $\Sigma$ is measurable with respect to the weak topology of $X$. Since $S(x, r)$ is closed in the weak topology, the set $\{\omega \in \Omega \mid \Sigma(\omega) \cap S(x, r) \neq \varnothing\}$ belongs to $\mathscr{A}$ for every $x \in X$ and $0<r<+\infty$. From this and the fact that every open (in the strong topology) set in $X$ is a countable union of closed balls (for $X$ separable) it follows that $\Sigma$ is weakly measurable with respect to the strong topology of $X$.

THEOREM 1. Let $\Omega$ be a measurable space. Let $X$ and $Y$ be separable Hilbert spaces and let $D$ be a subset of $X$. Then every random contraction $f: \Omega \times D \rightarrow Y$ can be extended to a random contraction defined on all $\Omega \times X$.

PROOF. First we show that $f$ can be extended to a random contraction $\tilde{f}: \Omega \times$ $\left(D \cup\left\{x_{0}\right\}\right) \rightarrow Y$, where $x_{0} \in X \backslash D$. For this, let $\left\{a_{i}\right\}$ be a dense countable subset of $D$. For $\omega \in \Omega$, set

$$
\Gamma(\omega)=\bigcap_{i=1}^{\infty} S\left(f\left(\omega, a_{i}\right),\left|a_{i}-x_{0}\right|\right) .
$$

We claim that for every $\omega \in \Omega, \Gamma(\omega) \neq \varnothing$. Otherwise we would have $\Gamma\left(\omega_{0}\right)=\varnothing$ for some $\omega_{0} \in \Omega$. Since $\left|f\left(\omega_{0}, a_{i}\right)-f\left(\omega_{0}, a_{j}\right)\right| \leq\left|a_{i}-a_{j}\right|$ and $\bigcap_{i=1}^{\infty} S\left(a_{i},\left|a_{i}-x_{0}\right|\right) \neq \varnothing$, property $(\mathrm{K})$ furnishes the contradiction.

By Lemma 1 the multifunction $\Gamma$ is weakly measurable and so, by [6], it admits a measurable selection, say $\gamma$. It is easy to see that a map $\tilde{f}: \Omega \times\left(D \cup\left\{x_{0}\right\}\right) \rightarrow Y$ given by $\tilde{f}(\omega, x)=f(\omega, x)$, if $(\omega, x) \in \Omega \times D$ and $\tilde{f}(\omega, x)=\gamma(\omega)$, if $(\omega, x) \in \Omega \times\left\{x_{0}\right\}$, is an admissible extension.

Now let $A$ be a dense countable subset of $X \backslash D$. By induction $f$ can be extended to a random contraction defined on $\Omega \times(D \cup A)$. Since $D \cup A$ is dense in $X$, by a standard argument $f$ can be extended to a random contraction defined on $\Omega \times X$.

Corollary 1. Let $\Omega, X, Y, D$ be as in Theorem 1 , let $f: \Omega \times D \rightarrow Y$ be such that for every $x \in D, f(\cdot, x)$ is measurable and, for every $\omega \in \Omega, f(\omega, \cdot)$ is Lipschitz with constant $L(\omega)$. In addition suppose that $L$ is a measurable function from $\Omega$ to $(0,+\infty)$. Then $f$ can be extended to a map $\tilde{f}: \Omega \times X \rightarrow Y$ such that for every $x \in X, \tilde{f}(\cdot, x)$ is measurable and, for every $\omega \in \Omega, \tilde{f}(\omega, \cdot)$ is Lipschitz with the same constant $L(\omega)$. 
COROLlaRY 2. Let $\Omega$ be a measurable space and let $Y$ be a separable Hilbert space. Let $f_{i}, g_{i}: \Omega \rightarrow Y(i \in \mathbf{N})$ be measurable functions such that $\mid g_{i}(\omega)-$ $g_{j}(\omega)|\leq| f_{i}(\omega)-f_{j}(\omega) \mid$ for every $\omega \in \Omega$ and every $i, j \in \mathbf{N}$. Let $f: \Omega \rightarrow Y$ be a measurable function. Then there exists a measurable function $g: \Omega \rightarrow Y$ such that $\left|g_{i}(\omega)-g(\omega)\right| \leq\left|f_{i}(\omega)-f(\omega)\right|$ for every $\omega \in \Omega$ and every $i \in \mathbf{N}$.

PROOF. By property $(\mathrm{K})$ and Lemma 1 we conclude that the multifunction $\Gamma: \Omega \rightarrow 2^{Y}$ given by $\Gamma(\omega)=\bigcap_{i=1}^{\infty} S\left(g_{i}(\omega),\left|f_{i}(\omega)-f(\omega)\right|\right)$ is well defined and weakly measurable. Thus it admits a measurable selection, say $g$. Clearly $g$ satisfies the statement of Corollary 2.

THEOREM 2. Let $\Omega$ be a complete measurable space. Let $X$ and $Y$ be separable metric spaces such that the pair $(X, Y)$ has the property $(\mathrm{K})$. Let $D$ be a proper subset of $X$. Then every random contraction $f: \Omega \times D \rightarrow Y$ can be extended to a random contraction defined on all $\Omega \times X$.

PROOF. Runs as that of Theorem 1. In the place of the multifunction given by (2) we consider the multifunction $\Gamma: \Omega \rightarrow 2^{X}$ given by

$$
\Gamma(\omega)=\bigcap_{i=1}^{\infty} S\left(f\left(\omega, a_{i}\right), d_{1}\left(a_{i}, x_{0}\right)\right) .
$$

The measurability of $\Gamma$ follows from [5, Theorem 3.5(iii)].

COROLlaRY 3. Let $X$ and $Y$ be normed spaces and let $\Omega$ and $D$ be as in Theorem 2. Suppose that the pair $(X, Y)$ has property $(\mathrm{K})$. Let $f: \Omega \times D \rightarrow Y$ be such that for every $x \in D, f(\cdot, x)$ is measurable and, for every $\omega \in \Omega, f(\omega, \cdot)$ is Lipschitz with constant $L(\omega)$. In addition suppose that $L$ is a measurable function from $\Omega$ to $\mathbf{R}$. Then $f$ can be extended to a map $\tilde{f}: \Omega \times X \rightarrow Y$ such that for every $x \in X, \tilde{f}(\cdot, x)$ is measurable and, for every $\omega \in \Omega, \tilde{f}(\omega, \cdot)$ is Lipschitz with the same constant $L(\omega)$.

Adopting the argument of the proof of [8, Theorem 11.3] and using the above results one can obtain the following

COROLlaRY 4. Let $\Omega$ be a complete measurable space. Let $X$ be a separable Hilbert space and let $D$ be a subset of $X$. Suppose that $f: \Omega \times D \rightarrow X$ is such that for every $x \in D, f(\cdot, x)$ is measurable and, for every $\omega \in \Omega, f(\omega, \cdot)$ is a Lipschitz-Hölder map of order $\alpha, 0<\alpha \leq 1$ (i.e. $\left.\left|f\left(\omega, x_{1}\right)-f\left(\omega, x_{2}\right)\right| \leq L(w)\left|x_{1}-x_{2}\right|^{\alpha}, x_{1}, x_{2} \in D\right)$. In addition suppose that $L$ is a measurable function from $\Omega$ to $(0,+\infty)$. Then $f$ can be extended to a map $\tilde{f}: \Omega \times X \rightarrow Y$ preserving measurability with respect to the first variable and the Lipschitz-Hölder condition with respect to the second variable with the same constant $L(\omega)$.

THEOREM 3. Let $\Omega$ be a measurable space. Let $X$ be a finite-dimensional Hilbert space and let $D$ be a closed subset of $X$. Let $f: \Omega \times D \rightarrow X$ be a random isometry (i.e. for every $x \in D, f(\cdot, x)$ is measurable and, for every $\omega \in \Omega, f(\omega, \cdot)$ is an isometry). Then $f$ can be extended to a random isometry defined on $\Omega \times X$.

ProOF. Fix $u \in D$ and set $\widehat{D}=D-u$. Consider the map $g: \Omega \times \widehat{D} \rightarrow X$ given by $g(\omega, x)=f(\omega, x+u)-f(\omega, u)$. Clearly $g$ is a random isometry. For every $\omega \in \Omega, g(\omega, 0)=0$, and so, by $[7], g(\omega, \cdot)$ is linear. Observe that $|g(\omega, x)|=|x|$ and 
$\langle g(\omega, x), g(\omega, y)\rangle=\langle x, y\rangle, \omega \in \Omega, x, y \in X$. (Here $|\cdot|$ stands for the norm and $\langle\cdot, \cdot\rangle$ for the inner product.) Let $V$ be the linear span of $\widehat{D}$. Define $\hat{g}: \Omega \times V \rightarrow X$ by

$$
\hat{g}\left(\omega, \sum_{i=1}^{n} \alpha_{i} x_{i}\right)=\sum_{i=1}^{n} \alpha_{i} g\left(\omega, x_{i}\right), \quad x_{1}, \ldots, x_{n} \in \widehat{D}, \alpha_{1}, \ldots, \alpha_{n} \in \mathbf{R} .
$$

Observe that $\hat{g}$ is a random isometry.

Consider the multifunction $\Gamma: \Omega \rightarrow 2^{X}$ given by $\Gamma(\omega)=\hat{g}(\omega, V)$. By [5, Theorem 6.5] $\Gamma$ is weakly measurable. Moreover, for every $\omega \in \Omega, \operatorname{dim} \Gamma(\omega)=\operatorname{dim} V$. Denote by $V^{\perp}$ and $\Gamma^{\perp}(\omega)$ the orthogonal complement of $V$ and $\Gamma(\omega)$, respectively. Clearly $\operatorname{dim} V^{\perp}=\operatorname{dim} \Gamma^{\perp}(\omega)$. Let $\left\{\gamma_{i}: i \in \mathbf{N}\right\}$ be a measurable representation of $\Gamma$ (see [1, Theorem III.6]). For $i \in \mathbf{N}$ consider the multifunction $\Gamma_{i}: \Omega \rightarrow 2^{X}$ given by $\Gamma_{i}(\omega)=$ $\left\{x \in X \mid\left\langle\gamma_{i}(\omega), x\right\rangle=0\right\}$. Since for any $y \in X, \operatorname{dist}\left(y, \Gamma_{i}(\omega)\right)=\left|\left\langle\gamma_{i}(\omega), y\right\rangle\right| /\left|\gamma_{i}(\omega)\right|$, it follows from [5, Theorem 3.3] that $\Gamma_{i}$ is weakly measurable. From the equality $\Gamma^{\perp}(\omega)=\bigcup_{n \geq 1}\left(\bigcap_{i \geq 1} \Gamma_{i}(\omega) \cap S(0, n)\right)$ and [5, Theorem 4.1 and Proposition 2.3] it follows that $\Gamma^{\perp}$ is measurable.

Let $\partial S$ denote the boundary of the unit ball $S$ of $X$, and let $k=\operatorname{dim} V^{\perp}$. By [5, Proposition 2.4] the multifunction $\Gamma^{\perp} \cap \partial S$ is measurable. Let $\xi_{1}: \Omega \rightarrow X$ be a measurable selection of $\Gamma^{\perp} \cap \partial S$. Define $\Sigma_{1}: \Omega \rightarrow 2^{X}$ by $\Sigma_{1}(\omega)=\{x \in$ $\left.X \mid\left\langle x, \xi_{1}(\omega)\right\rangle=0\right\}$. The above argument shows that $\Sigma_{1}$ is measurable. By [5, Theorem 4.1] the multifunction $\Gamma^{\perp} \cap \Sigma_{1} \cap \partial S$ is measurable. Let $\xi_{2}$ be a measurable selection of $\Gamma^{\perp} \cap \Sigma_{1} \cap \partial S$. By recurrence one can construct functions $\xi_{2}, \ldots, \xi_{k}$ such that $\xi_{i}(i=2, \ldots, k)$ is a measurable selection of $\Gamma^{\perp} \cap \Sigma_{1} \cap \cdots \cap \Sigma_{i-1} \cap \partial S$. (Here $\Sigma_{i}(\omega)=\left\{x \in X \mid\left\langle x, \xi_{i}(\omega)\right\rangle=0\right\}$.) Note that for every fixed $\omega \in \Omega$, the vectors $\xi_{1}(\omega), \ldots, \xi_{k}(\omega)$ are orthonormal.

Let $e_{1}, \ldots, e_{k}$ be an orthonormal base $V^{\perp}$. Clearly the function $h: \Omega \times\left\{e_{1}, \ldots\right.$, $\left.e_{k}\right\} \rightarrow X$ given by $h\left(\omega, e_{i}\right)=\xi_{i}(\omega)$ is a random isometry. As above, $h$ can be extended to all $\Omega \times V^{\perp}$. Since for every $(\omega, x) \in \Omega \times V^{\perp}, h(\omega, x) \in \Gamma^{\perp}(\omega)$, there is a random linear isometry $\tilde{g}$ of all $\Omega \times X$ onto $X$ that extends $g$ (cf. [4, Theorem 3]). To finish the proof it suffices to observe that the random isometry $\tilde{f}: \Omega \times X \rightarrow Y$ given by $\tilde{f}(\omega, x)=\tilde{g}(\omega, x-u)+f(\omega, u)$ is an admissible extension.

REMARK. Theorem 3 fails, even in deterministic case, if $X$ is supposed to be infinite-dimensional (see [8]).

The next theorem is a random version of Grunbaum's result [2] concerning the properties of monotone sets.

THEOREM 4. Let $\Omega$ be a measurable space and let $Y$ be a separable Hilbert space. Let $f_{i}, g_{i}: \Omega \rightarrow Y(i=1, \ldots, n)$ be measurable functions such that

$$
\left\langle f_{i}(\omega)-f_{j}(\omega), g_{i}(\omega)-g_{j}(\omega)\right\rangle \geq 0, \quad \omega \in \Omega, i, j=1, \ldots, n .
$$

Let $\rho: \Omega \rightarrow(0,+\infty)$ be a measurable function.

Then there is a measurable function $h: \Omega \rightarrow Y$ such that

$$
\left\langle f_{i}(\omega)+\rho(\omega) h(\omega), g_{i}(\omega)-h(\omega)\right\rangle \geq 0, \quad \omega \in \Omega, i=1, \ldots, n .
$$

PROOF. From [2] it follows that for every fixed $\omega \in \Omega$, there is a vector $u \in Y$ (more precisely $u \in \operatorname{span}\left\{f_{1}(\omega), \ldots, f_{n}(\omega), g_{1}(\omega), \ldots, g_{n}(\omega)\right\}$ ) such that

$$
\left\langle f_{i}(\omega)+\rho(\omega) u, g_{i}(\omega)-u\right\rangle \geq 0, \quad i=1,2, \ldots, n .
$$


A simple calculation shows that for every vector $u$ satisfying (3) we have

$$
|u| \leq \sigma(\omega)
$$

where $\sigma(\omega)=\left(\beta(\omega)+\sqrt{\beta^{2}(\omega)+4 \alpha(\omega) \rho(\omega)}\right) / 2 \rho(\omega), \alpha(\omega)=\sup \left\{\left|\left\langle f_{i}(\omega), g_{i}(\omega)\right\rangle\right|\right.$ : $i=1,2, \ldots, n\}, \beta(\omega)=\sup \left\{\left|\rho(\omega) g_{i}(\omega)-f_{i}(\omega)\right|: i=1,2, \ldots, n\right\}$. Note that a multifunction $\Sigma: \Omega \rightarrow 2^{Y}$ given by $\Sigma(\omega)=S(0, \sigma(\omega))$ is weakly measurable, for $\sigma(\cdot)$ being measurable.

For $i=1,2, \ldots, n$ and $k \in \mathbf{N}$ consider $\Gamma_{i}^{k}: \Omega \rightarrow 2^{Y}$ given by

$$
\Gamma_{i}^{k}(\omega)=\operatorname{cl}\left\{y \in Y \mid\left\langle f_{i}(\omega)+\rho(\omega) y, g_{i}(\omega)-y\right\rangle>-1 / k\right\} .
$$

(Here $\operatorname{cl}\{\cdots\}$ denotes the closure of the set $\{\cdots\}$.) By virtue of $[\mathbf{2}$ and $\mathbf{5}$, Theorem 6.2 and Proposition 2.6], $\Gamma_{i}^{k}$ is nonempty valued and weakly measurable. Consider the multifunction $\Gamma: \Omega \rightarrow 2^{Y}$ given by

$$
\Gamma(\omega)=\Sigma(\omega) \cap \bigcap_{\substack{i=1, \ldots, n \\ k \in \mathbf{N}}} \Gamma_{i}^{k}(\omega) .
$$

From [2] and (4) it follows that $\Gamma$ is nonempty valued. Since $\Sigma$ and $\Gamma_{i}^{k}$ are weakly measurable with respect to the weak topology of $Y$ and, in addition, $\Sigma$ is weakly compact valued, by [5, Theorem 4.1] $\Gamma$ is measurable with respect to the weak topology of $Y$, and so weakly measurable with respect to the strong topology of $Y$, $Y$ being separable. Let $h$ be a measurable selection of $\Gamma$. It is easy to see that $h$ satisfies the statement of Theorem 4.

3. Acknowledgement. The authors wish to thank the referee for extremely attentive reading and useful critical remarks.

\section{REFERENCES}

1. C. Castaing and M. Valadier, Convex analysis and measurable multifunctions, Lecture Notes in Math., vol. 580, Springer-Verlag, Berlin and New York, 1977.

2. B. Grunbaum, A generalization of theorems of Kirszbraun and Minty, Proc. Amer. Math. Soc. 13 (1962), 812-814.

3. L. Danzer, B. Grunbaum and V. Klee, Helly's theorems and its relatives, Amer Math. Soc. Proc. Sympos. Pure Math., vol. 7, Amer. Math. Soc., Providence, R. I., 1963, pp. 101-180.

4. O. Hans, Measurability of extensions of continuous random transforms, Ann. Math. Statist. 30 (1959), 1152-1157.

5. C. J. Himmelberg, Measurable relations, Fund. Math. 87 (1975), 53-72.

6. K. Kuratowski and C. Ryll-Nardzewski, A general theorem on selectors, Bull. Acad. Polon. Sci. Math. Astronom. Phys. 13 (1965), 397-403.

7. S. Mazur and S. Ulam, Sur les transformations isométriques d'espace vectoriels normés, C. R. Acad. Sci. Paris 194 (1932), 946-948.

8. J. H. Wells and L. R. Williams, Embeddings and extensions in analysis, Ergebnissse Math. Grenz. 84 (1975)

9. F. Valentine, A Lipschitz condition preserving extensions for a vector function, Amer. J. Math. 67 (1945), 83-93.

\section{SISSA, Strada Costiera 11, 34014 Trieste, Italy}

Zaklad Statystyki Matematycznej AR, Al. Mickiewicza 21, Kraków, Poland

Current address (J. Myjak): Facoltà di Ingegneria, Università dell'Aquila, 67040 Roio Poggio (AQ), Italy 